\title{
Enhanced therapeutic efficacy of adenovirus-mediated interleukin-24 gene therapy combined with ionizing radiotherapy for nasopharyngeal carcinoma
}

\author{
JISHENG LIU $^{1 *}$, YUJUAN ZHANG $^{2 *}$, PENG SUN $^{1}$, YUFENG XIE $^{3,4}$, JIM XIANG $^{4}$ and JICHENG YANG $^{3}$ \\ ${ }^{1}$ Department of ENT, The First Affiliated Hospital of Soochow University; ${ }^{2}$ Institute for Fetal Origin Diseases and \\ Reproductive Medicine Center, The First Affiliated Hospital and Experiment Center of Soochow University; \\ ${ }^{3}$ Cell and Molecular Biology Institute, College of Medicine, Soochow University, Suzhou, P.R. China; \\ ${ }^{4}$ Department of Oncology and Immunology, University of Saskatchewan, Saskatoon, Saskatchewan, Canada
}

Received October 6, 2012; Accepted May 15, 2013

DOI: 10.3892/or.2013.2550

\begin{abstract}
Melanoma differentiation-associated gene-7 (mda-7)/interleukin-24 (IL-24), a unique cytokine tumor suppressor, displays ubiquitous antitumor activities and cancerspecific cytotoxicities via multiple signaling pathways. In the present study, we investigated the antitumor effect of adenovirus-mediated IL-24 (AdVIL-24) gene therapy in conjunction with ionizing radiation on $\mathrm{CNE}-2 \mathrm{Z}$ human nasopharyngeal carcinoma (NPC) cells in vitro and in vivo in athymic nude mice, and its potential mechanisms. We demonstrated that AdVIL-24 gene therapy plus ionizing radiotherapy induced enhanced growth inhibition, cell cycle G1 phase arrest and apoptosis in vitro in CNE-2Z human NPC cells and in vivo in CNE-2Z xenografted tumors subcutaneously implanted in athymic nude mice. Mechanistically, AdVIL-24 combined with ionizing radiation led to the substantial upregulation of P21 and P27 cyclin-dependent kinase (CDK) inhibitors, ratio of pro-apoptotic to anti-apoptotic molecules Bax/Bcl-2 and cleaved caspase-3 as well as downregulation of cyclin $\mathrm{E}$ and CDK2 in vitro and in vivo in CNE-2Z human NPC cells. Furthermore, AdVIL-24 plus radiation additively reduced the tumor vessel CD34 expression and microvessel density in vivo. More importantly, AdVIL-24 potentially blocked the radiation-induced enhancement of vascular endothelial growth factor (VEGF), a pro-angiogenic factor. The enhanced antitumor activity against NPC elicited by AdVIL-24 gene therapy combined with ionizing radiotherapy was closely associated with the enhanced induction of G1 phase arrest
\end{abstract}

Correspondence to: Professor Jicheng Yang, Cell and Molecular Biology Institute, College of Medicine, Soochow University, Suzhou 215123, P.R. China

E-mail: jcyang@suda.edu.cn

*Contributed equally

Key words: interleukin-24, gene therapy, ionizing radiotherapy, nasopharyngeal carcinoma, enhanced antitumor effect and apoptosis via additive modulation of cell cycle regulatory molecules and activation of intrinsic apoptotic pathways, and the overlapping inhibition of tumor angiogenesis. Thus, our results suggest that AdVIL-24 gene therapy combined with ionizing radiotherapy may be a novel and effective treatment strategy for human NPC.

\section{Introduction}

Nasopharyngeal carcinoma (NPC) is a squamous cell carcinoma originating from epithelial cells of the nasopharynx, which is a distinctive type of head and neck cancer (HNC) (1). Genetic susceptibility and environmental factors such as Epstein-Barr virus (EBV) infection are major causes of NPC $(1,2)$. The neoplasm is endemic in Southern China and Southeast Asia $(3,4)$, representing a marked ethnic and geographic distribution. The annual incidence rate of NPC still remains very high and peaks at 50 cases per 100,000 people in the prevalent regions (3). NPC has the highest metastatic potential among HNC. The majority of patients have metastasis to regional lymph nodes or even distant organs at the time of diagnosis (5). The conventional treatments for NPC involve radiotherapy, chemotherapy and the concurrent combination. However, the 5-year overall survival (OS) rate after treatment is $70 \%$ (6) and has not been significantly improved. Moreover, 30 to $40 \%$ patients eventually will relapse with locoregional recurrence and distant metastasis, with poor median survival ranging from 7.2 to 22 months $(7,8)$. Hence, the search for novel therapeutic modalities to treat patients with metastatic and recurrent NPC is urgently needed. The intensive multimodality management of standard and novel therapeutic agents may improve the prognosis of NPC.

Melanoma differentiation-associated gene-7 (mda-7)/interleukin-24 (IL-24) is a unique member of the IL-10 family (9), exhibiting ubiquitous antitumor activities and cancer-specific cytotoxicities (10). IL-24 can strongly suppress tumor growth irrespective of p53 status via induction of apoptosis and cell cycle arrest (10). IL-24 can also inhibit tumor angiogenesis through direct suppression of vascular endothelial cell differentiation and migration via interacting with the 
IL-22R1/IL-20R2 heterodimeric receptor on vascular endothelial cells (11), and indirectly downregulating the production of vascular endothelial growth factor (VEGF) and IL-8 pro-angiogenic factors (12). Furthermore, IL-24 as a pro-Th1 cytokine can display potent immunoactivating property and enhance antitumor immunity by stimulating the production of secondary cytokines such as IL-6, tumor necrosis factor (TNF) $-\alpha$ and interferon (IFN)- $\gamma$ (13). In addition, IL-24 can suppress tumor cell invasion and metastasis by reduction of focal adhesion kinase (FAK) and matrix metalloproteinase (MMP)-2 and MMP-9 (14). Interestingly, IL-24 can induce cytotoxic autophagy in tumor cells through endoplasmic reticulum (ER) stress response and protein kinase R (PKR)-like ER kinase (PERK) activation $(15,16)$. Notably, IL-24 can sensitize radiotherapy or chemotherapy of tumors and exert profound bystander antitumor activities, leading to the augmentation of antitumor efficacy (10). Thus, IL-24 as a promising tumor suppressor has been hailed as a 'magic bullet' for cancer.

Gene therapy represents a novel therapeutic strategy against cancer including NPC (17), and is based on the introduction of genetic material such as a tumor-suppressor gene and small short hairpin RNA (shRNA) targeting an oncogene or a pro-angiogenic factor gene into tumor cells. The combination of gene therapy and conventional radiotherapy or chemotherapy can improve antitumor benefits and reduce side-effects $(18,19)$. IL-24 has been shown to exhibit striking radiosensitizing effects in a diverse spectrum of tumors $(12,20,21)$. We previously demonstrated that adenovirus-mediated IL-24 (AdVIL-24) gene therapy can elicit potential antitumor activity in human laryngocarcinoma (22). However, the therapeutic effect of AdVIL-24 alone or combined with ionizing radiotherapy on human NPC is still elusive. In the present study, we investigated the therapeutic efficacy of AdVIL-24 gene therapy combined with ionizing radiation in vitro in CNE-2Z human NPC (nonkeratinizing carcinoma) cells and in vivo in an athymic nude mouse CNE-2Z human NPC xenografted tumor model, and we also elucidated the underlying mechanisms.

\section{Materials and methods}

Adenoviruses, cell lines, reagents and mice. The recombinant replication-incompetent Ad5E1- and E3-deleted adenovirus AdVIL-24/green fluorescent protein (GFP) (termed AdVIL-24) expressing both human IL-24 and GFP and its control blank adenovirus AdVGFP (termed AdV) expressing GFP but no human IL-24 were constructed in our laboratory (23), at the Cell and Molecular Biology Institute, College of Medicine, Suzhou University (Suzhou, Jiangsu, China). The CNE-2Z human NPC cell line was purchased from the American Type Culture Collection (ATCC, Rockville, MD, USA). The CNE-2Z tumor cells were cultured in RPMI-1640 (Invitrogen, Shanghai, China) supplemented with $10 \%$ fetal bovine serum (FBS; HyClone, Logan, UT, USA), respectively. The TRIzol and reverse transcriptase polymerase MuMLV were purchased from Invitrogen. The DL2000 DNA marker was purchased from Takara (Shanghai, China). The 3-(4,5-dimethylthiazol-2-yl)2,5-diphenyltetrazolium bromide (MTT) kit and mammalian cell lysis kit were purchased from Sigma (Shanghai, China). The propidium iodide (PI) cell cycle detection kit and
Annexin-PE/7-AAD apoptosis detection kit were purchased from Nanjing Kaiji Biological Engineering Co., Ltd. (Nanjing, Jiangsu, China). The antibodies specific for IL-24, P21, P27, cyclin E, cyclin-dependent kinase 2 (CDK2), Bcl-2, Bax, caspase-3/cleaved caspase-3, $\beta$-actin, CD34 and VEGF were purchased from Abcam (Shanghai, China). The SuperEnhanced chemiluminescence detection kit was purchased from Applygen Technologies, Inc. (Beijing, China). The UltraSensitive ${ }^{\mathrm{TM}} \mathrm{SP}$ kit was purchased from Maixin (Fuzhou, Fujian, China). The terminal deoxynucleotidyl transferase-mediated dUTP nick end labeling (TUNEL) apoptosis detection kit was purchased from Beyotime Institute of Biotechnology (Beijing, China). Male athymic BALB/c nude mice (4- to 6-week-old) were purchased from the Shanghai Experimental Animal Center (Shanghai, China) and maintained in the animal facility at Soochow University according to the Animal Research Committee Guidelines of Soochow University.

AdVIL-24 gene transfer. To evaluate the optimal multiplicity of infection (MOI) for a maximal adenoviral infection and human IL-24 transgene expression in CNE-2Z tumor cells, the CNE-2Z human NPC cells were infected with recombinant adenovirus AdVIL-24 expressing both human IL-24 and marker GFP or control blank adenovirus AdV expressing GFP at various MOIs $(0,10,25,50,100$ and 200), respectively. The GFP expression and adenoviral infection efficiency were then examined by fluorescence microscopy. Moreover, adenovirusdirected human IL-24 transgene expression in CNE-2Z NPC cells was determined by reverse transcription (RT)-PCR and western blot analysis as described previously (24).

Flow cytometric analysis. To select an optimal irradiation dose in the in vitro combined experiment of AdVIL-24 gene therapy and ionizing radiotherapy for CNE-2Z tumor cells, the CNE-2Z human NPC cells were cultured in T25 flasks at $1 \times 10^{6}$ cells/flask. Forty-eight hours later, the tumor cells were irradiated at various doses $\left(2,4,6\right.$ and 8 Gy) using a ${ }^{60} \mathrm{Co}-\gamma$ source $(1 \mathrm{~Gy} / \mathrm{min})$. After another $24-\mathrm{h}$ incubation, the irradiated and unirradiated CNE-2Z tumor cells were harvested, washed with cold PBS and then subjected to apoptosis analysis by flow cytometry using Annexin V-PE (early apoptotic marker) and 7-AAD (late apoptotic marker) double staining following the manufacturer's instructions. Briefly, the tumor cells $\left(1 \times 10^{5}\right)$ were incubated in the presence of $5 \mu \mathrm{l}$ Annexin V-PE and $5 \mu \mathrm{l}$ 7-AAD in $100 \mu \mathrm{l}$ of $1 \mathrm{X}$ Annexin $\mathrm{V}$ binding buffer at room temperature. After a 15-min incubation, $400 \mu \mathrm{l}$ of $1 \mathrm{X}$ binding buffer was added and the apoptotic cells were then analyzed by flow cytometry. To further assess the combined effect of AdVIL-24 plus radiotherapy on cell cycle profiles and apoptosis of CNE-2Z tumor cells, the CNE-2Z human NPC cells were also cultured in $\mathrm{T} 25$ flasks at $1 \times 10^{6}$ cells/flask. After a 24-h incubation, the CNE-2Z tumor cells were infected with AdVIL-24 or AdV used as a blank adenovirus control at the MOI of 100. The medium containing PBS without the adenovirus was used as a cell control (PBS control). Twenty-four hours post adenoviral infection, the tumor cells were further exposed to radiation at the optimal dose of $4 \mathrm{~Gy}$. The experiments were divided into 5 groups: PBS, AdV, AdVIL-24 alone, 4 Gy alone and AdVIL-24 plus 4 Gy. Twenty-four hours after irradiation, tumor cells were then processed to analyze apop- 
tosis by flow cytometry as described above. In addition, the cell cycle distribution of treated and untreated CNE-2Z tumor cells was determined using PI staining by flow cytometric analysis. In brief, these cells were harvested and washed in cold PBS. The cell pellets were fixed in $70 \%$ cold alcohol for more than $24 \mathrm{~h}$ at $4^{\circ} \mathrm{C}$, washed in cold PBS, stained with PI solution [50 $\mu \mathrm{g} / \mathrm{ml} \mathrm{PI}$, $50 \mu \mathrm{g} / \mathrm{ml}$ RNase A and $0.1 \%(\mathrm{v} / \mathrm{v})$ Triton X-100] at $4^{\circ} \mathrm{C}$ in the dark for $30 \mathrm{~min}$, then washed and analyzed by flow cytometry.

MTT assay. The in vitro suppressive effect of AdVIL-24 plus ionizing radiation on CNE-2Z human NPC cells was determined by MTT assay. The CNE-2Z tumor cells were dispensed into 96-well culture plates at $1 \times 10^{4}$ cells/well. After a 24-h incubation, the CNE-2Z tumor cells were infected with 100 MOI AdVIL-24 or AdV or without the adenovirus (PBS control) followed by irradiation with an optimal dose of $4 \mathrm{~Gy}$ at day 1 after infection. Before treatment and at different time points after single and/or combined treatment, the viability of CNE-2Z tumor cells was then analyzed using the MTT kit according to the manufacturer's protocol.

Clonogenic survival assay. The in vitro inhibitory effect of AdVIL-24 plus ionizing radiation on CNE-2Z human NPC cells was also assessed by clonogenic survival assay. Briefly, $24 \mathrm{~h}$ after infection, the $100 \mathrm{MOI}$ AdVIL-24- and AdV-infected CNE-2Z tumor cells or uninfected control CNE-2Z tumor cells were dispensed into 6-well culture plates at 200 cells/well, and then irradiated with $4 \mathrm{~Gy}$. After another 2 weeks of culture, the cells were washed with PBS, fixed with methanol and stained with $0.1 \%$ crystal violet. The number of colonies was then manually counted under a microscope. The colonies consisting of 50 or more cells were considered to be survivors.

Western blot analysis. The CNE-2Z human NPC cells were treated with PBS, AdV (100 MOI), AdVIL-24 (100 MOI) alone, 4 Gy alone and AdVIL-24 (100 MOI) plus 4 Gy as described above. Twenty-four hours after irradiation, these cells were collected, washed with cold PBS and lysed in lysis buffer $\left(1 \times 10^{7}\right.$ cells $/ 1 \mathrm{ml}$ lysis buffer) for preparation of total cellular lysate using the mammalian cell lysis kit. The protein concentration was determined by BCA protein assay using a spectrophotometer. The total cellular lysates (100 $\mu \mathrm{g} / \mathrm{lane})$ were resolved by $12 \%$ sodium dodecyl sulfate-polyacrylamide gel electrophoresis (SDS-PAGE) and subsequently transferred onto a polyvinylidene difluoride (PVDF) membrane for western blot analysis using a panel of antibodies specific for P21, P27, cyclin E, CDK2, Bcl-2, Bax and caspase-3 (pro-caspase-3 and cleaved caspase-3), respectively. The membrane was then washed and developed using the SuperEnhanced chemiluminescence detection kit. The protein bands were visualized after exposure of the membranes to Kodak X-ray film.

Real-time quantitative RT-PCR analysis. The in vitro expression of P21, P27, cyclin E, CDK2, Bcl-2 and Bax in the treated and untreated CNE-2Z human NPC cells was confirmed by SYBR-Green I-based real-time quantitative RT-PCR analysis using the following primers: P21-F, 5'-ccc gtg agc gat gga ac-3' and P21-R, 5'-aaa tct gtc atg ctg gtc tgc-3'; P27-F, 5'-gtc taa cgg gag ccc gag cet gg-3' and P27-R, 5'-gaa ggc cgg gtt ctt ctt ggg cg-3'; cyclin E-F, 5'-tgg cgt tta agt ccc ctg ac-3' and cyclin E-R, 5'-tca gtt ttg agc tcc ccg tc-3'; CDK2-F, 5'-ctc cag ggc cta get ttc tg-3' and CDK2-R, 5'-ttc agg agc tcg gta cca ca-3'; Bcl-2-F, 5'-tgt ggc ctt ctt tga gtt cg-3' and Bcl-2-R, 5'-cta ccc agc ctc cgt tat cc-3'; Bax-F, 5'-gga tgc gtc cac caa gaa-3' and Bax-R, 5'-gca ctc ccg cca caa aga-3'; and $\beta$-actin-F, $5^{\prime}$-tgc gtg aca tta agg aga ag-3' and $\beta$-actin-R, 5'-ctg cat cet gtc ggc aat g-3'. The cDNA quantities were normalized to the internal control gene $\beta$-actin measured in the same samples. Relative gene expression was calculated using the pooled cDNA from all samples by the $2^{-\Delta \Delta \mathrm{CT}}$ method as described previously $(25,26)$. The authenticity of PCR products was verified by melting curve analysis and agarose gel electrophoresis. Each sample was analyzed in triplicate in an independent reaction and the experiment was repeated three times.

Animal studies. The male athymic BALB/c nude mice were subcutaneously (s.c.) inoculated in the armpit of the right anterior limb with $2 \times 10^{6} \mathrm{CNE}-2 \mathrm{Z}$ human NPC cells and then monitored daily for tumor growth. Tumor volume was measured with a caliper and calculated using the formula, Tumor size $=a b^{2} / 2$, where $a$ is the larger and $b$ is the smaller of the two dimensions. When tumors grew to a mean tumor volume of $\sim 100 \mathrm{~mm}^{3}$, the CNE-2Z human NPC s.c. xenografted tumorbearing mice were subjected to AdVIL-24 human gene therapy by multi-point intratumoral injection of AdVIL-24 (1x10 $\left.{ }^{8}\right), \mathrm{AdV}$ $\left(1 \times 10^{8}\right)$ or PBS every other day for a total of 6 times, respectively. Following the second AdVIL-24 gene therapy, the mice bearing CNE-2Z xenografted tumors were further assigned to receive single radiotherapy at a dose of $10 \mathrm{~Gy} /$ tumor using a ${ }^{60} \mathrm{Co}-\gamma$ source $(1 \mathrm{~Gy} / \mathrm{min})$ as reported previously (27). All the mice for radiation were anesthetized with $10 \%$ chloral hydrate ( $3 \mu \mathrm{l} / \mathrm{g}$ body weight) and positioned in the radiation field so that only the tumor xenograft implanted on the armpit of the right anterior limb was exposed to the irradiation beam and the rest of the mouse's body was shielded by a lead block. Tumor progression and regression were monitored, and the tumor volume was measured daily. In addition, the tumor-bearing mice were sacrificed 2 weeks after treatment, and the CNE-2Z human NPC s.c. xenografted tumors were removed, weighed, fixed with $10 \%$ neutral formalin and embedded in paraffin for hematoxylin and eosin (H\&E) staining and immunohistochemistry analysis.

Immunohistochemistry. The in vivo expression of P21, P27, cyclin E, CDK2, Bcl-2, Bax, cleaved caspase-3, CD34 and VEGF in the treated and untreated CNE-2Z human NPC s.c. xenografted tumors was examined by immunohistochemistry using the UltraSensitive ${ }^{\mathrm{TM}}$ SP kit following the manufacturer's instructions. The presence of buffy or brown diaminobenzidine precipitates is indicative of positive reactivity. The integral optical density (IOD) of immunohistochemical intensity was analyzed by Image-Pro Plus 6.0 software (Media Cybernetics, Bethesda, MD, USA). In addition, the microvessel density (MVD) was assessed by CD34 immunostaining as described previously (28). Any endothelial cell cluster immunoreactive for CD34 clearly separated from adjacent microvessels was considered as a single countable vessel. To detect the apoptotic cells in vivo in the CNE-2Z human NPC xenografted tumors, tumor sections were further analyzed for apoptosis using the TUNEL apoptosis detection kit according to the manufacturer's protocols. Each value represents IOD, microvessels 


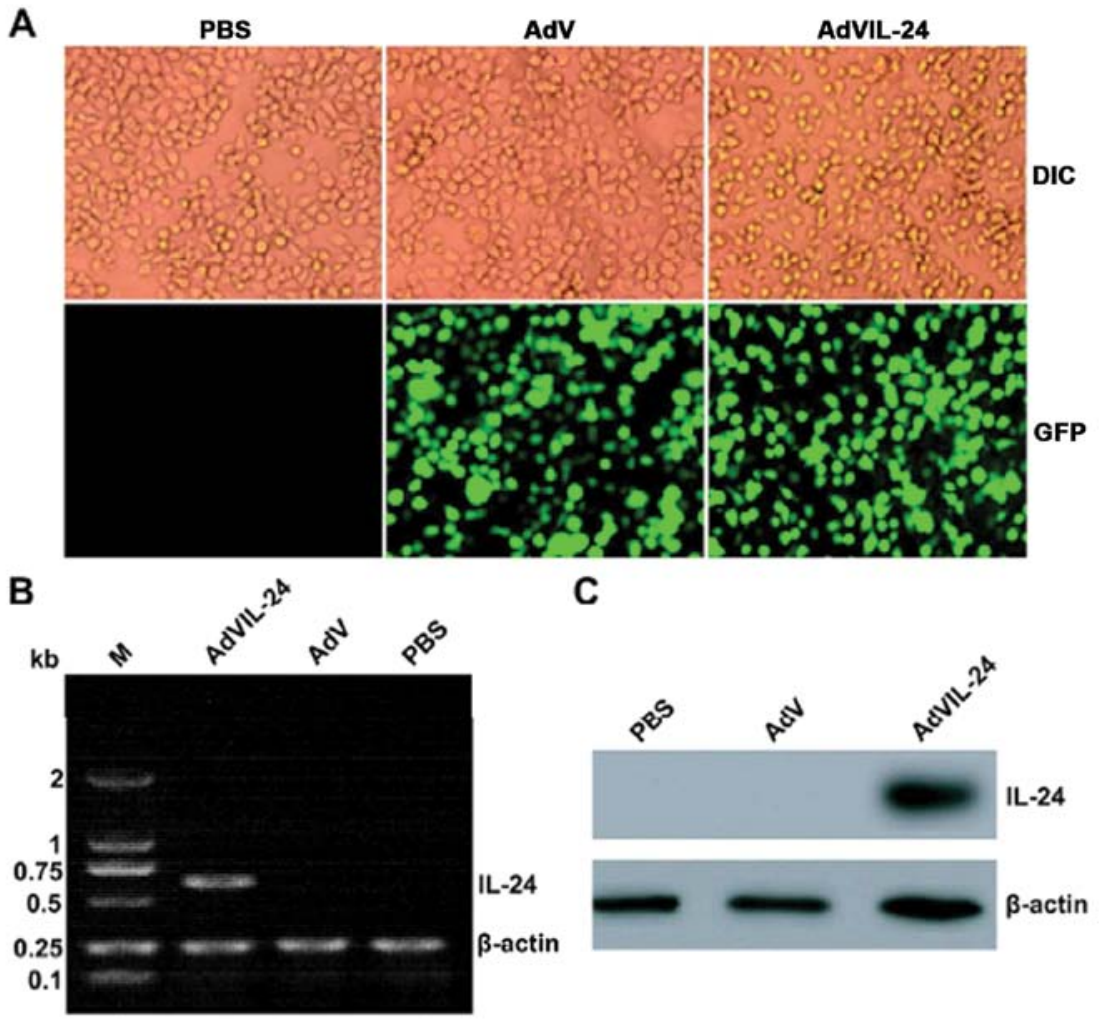

Figure 1. Adenovirus-mediated interleukin-24 (AdVIL-24) transgene expression. (A) Green fluorescent protein (GFP) expression and infection efficiency examined under fluorescence microscopy. The CNE-2Z human NPC cells were infected with AdVIL-24 or AdV at the optimal multiplicity of infection of 100 for $48 \mathrm{~h}$ and assessed under GFP fluorescence and differential interference contrast images by fluorescence microscopy. (B) AdVIL-24 transcriptional expression by RT-PCR analysis. Total cellular RNAs were extracted from AdVIL-24- or AdV-infected and uninfected CNE-2Z tumor cells. The first-strand cDNAs were synthesized from RNAs using reverse transcriptase; PCRs were conducted using primer sets specific for human IL-24 and housekeeping gene $\beta$-actin (an internal control), respectively. M, DL2000 DNA marker. (C) AdVIL-24 translational expression by western blot analysis. Total cellular lysates derived from AdVIL-24- or AdV-infected CNE-2Z tumor cells and uninfected control cells were analyzed by immunoblotting with anti-IL-24 and anti- $\beta$-actin (an internal control) antibody. Data shown are representative of three independent experiments.

or apoptotic cells counted at a high-power view (x200) by microscopy. The mean value represents the average number derived from five high-power fields of each case.

Statistical analysis and evaluation of combinatorial interaction. All data are presented as the mean \pm standard deviation (SD). The significance of the difference between groups was evaluated by one-way or two-way repeated measure analysis of variance (ANOVA) and multiple comparisons with SPSS 10.0 software (SPSS, Chicago, IL, USA). A value of $\mathrm{P}<0.05$ was considered statistically significant. The interactive effects of AdVIL-24 and radiotherapy were evaluated by Q-value (29), $\mathrm{Q}=\mathrm{F}(\mathrm{A}+\mathrm{B}) / \mathrm{FA}+(1-\mathrm{FA}) \mathrm{FB}$, where $\mathrm{F}(\mathrm{A}+\mathrm{B})$ represents the fraction affected by combined treatment with AdVIL-24 plus radiotherapy compared to the untreated control group, FA represents the fraction affected by AdVIL-24 alone and FB represents the fraction affected by radiotherapy alone. A value of $Q>1.15$ indicates a synergistic effect between AdVIL-24 and radiotherapy, $\mathrm{Q}<0.85$ indicates an antagonistic effect and $\mathrm{Q}$ between 0.85 and 1.15 indicates an additive effect.

\section{Results}

Transgene IL-24 overexpression. To assess the optimal MOI for a maximal adenovirus-directed IL-24 transgene expres- sion with minimal adenovirus itself-induced cytotoxic effect, CNE-2Z human NPC cells were infected with AdVIL-24 or $\mathrm{AdV}$ at various MOIs, and then examined under fluorescence microscopy. As shown in Fig. 1A, more than $90 \%$ of GFP expression was observed in the CNE-2Z tumor cells treated with AdVIL-24 or AdV at a MOI of 100 or above (data not shown), whereas the GFP expression was not found in the untreated control CNE-2Z tumor cells. In addition, there was almost no adenovirus-elicited cytotoxicity in the $100 \mathrm{MOI}$ blank AdV-infected CNE-2Z tumor cells (Fig. 1A). Therefore, we selected a MOI of 100 as an optimal dose for the adenoviral infection of the CNE-2Z tumor cell line in the following experiments. To further determine AdVIL-24 expression, the AdVIL-24- or AdV-treated and untreated CNE-2Z tumor cells were subjected to RT-PCR (Fig. 1B) and western blotting (Fig. 1C) analysis, respectively. These results indicated that exogenous IL-24 transgene mediated by the adenoviral transfer was efficiently expressed in the AdVIL-24-infected CNE-2Z tumor cells at both the transcriptional and translational levels.

Enhanced tumor suppression by AdVIL-24 plus radiation. Before performing experiments involving a combination of AdVIL-24 gene therapy plus ionizing radiotherapy, we first carried out a radiation dose-assessing study in vitro in the CNE-2Z human NPC cells by flow cytometric analysis of 
A
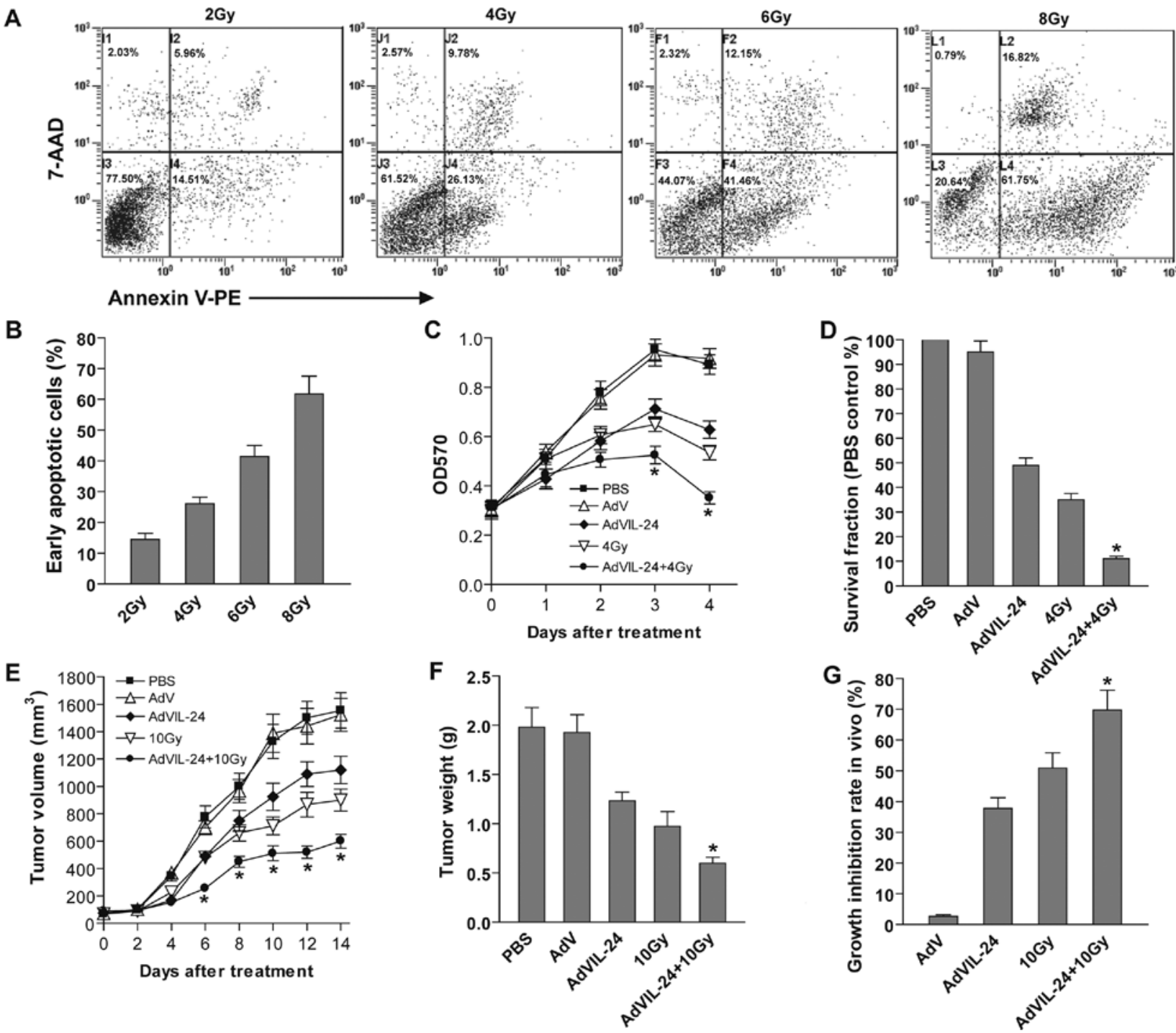

Figure 2. Adenovirus-mediated interleukin-24 (AdVIL-24) plus radiotherapy induces enhanced tumor suppression. (A and B) The effect of radiation alone on CNE-2Z tumor cells by flow cytometry. The CNE-2Z human NPC cells were irradiated with various doses of radiation. (A) Twenty-four hours later, the tumor cells were harvested, stained with Annexin V-PE and 7-AAD and then analyzed by flow cytometry. (B) The Annexin V single-positive cells in the total cell population represented early apoptotic cells. (C and D) AdVIL-24 combined with radiation induces enhanced growth inhibition in vitro. The CNE-2Z human NPC cells were treated with PBS, AdV, AdVIL-24, 4 Gy or AdVIL-24 plus 4 Gy for the indicated time periods. (C) The surviving cells were evaluated at day 0, 1 (i.e. $24 \mathrm{~h}$ after AdVIL-24 infection), 2 (i.e. $24 \mathrm{~h}$ after 4 Gy irradiation), 3 and 4 after treatment by MTT assay. "P<0.05 compared with AdVIL-24 and 4 Gy group; $\mathrm{Q}=0.916$ and 1.050, at day 3 and 4 after treatment, respectively, two-way repeated measure ANOVA and multiple comparisons, $\mathrm{n}=4$ replicates/condition. (D) The survival fraction in the clonogenic survival assay was calculated by comparison with the PBS-treated control group. "P $<0.05$ compared with AdVIL-24 and 4 Gy group; $\mathrm{Q}=1.074$, one-way repeated measure ANOVA and multiple comparisons, $\mathrm{n}=3$ replicates/condition. (E-G) AdVIL-24 combined with radiation induces enhanced growth inhibition in vivo. The athymic nude mice bearing CNE-2Z human NPC subcutaneously xenografted tumors were intratumorally injected with AdVIL-24, AdV or PBS every other day for a total of 6 times, respectively. The mice were further subjected to single radiotherapy at a dose of $10 \mathrm{~Gy} /$ tumor at day 2 post AdVIL-24 gene therapy (i.e. after the second AdVIL-24 gene therapy). (E) Tumor volume before and after treatment, and (F) tumor weight of the CNE-2Z xenografted tumors removed 14 days after treatment were measured. (G) Tumor growth inhibition rate in vivo was further calculated according to tumor weight. "P<0.05 compared with AdVIL-24 and $10 \mathrm{~Gy}$ group; $\mathrm{Q}_{\text {volume }}=1.099,1.098,0.981,1.127$ and 1.053 , at day $6,8,10,12$ and 14 after treatment, and $\mathrm{Q}_{\text {weight }}=1.004$, respectively; two-way and one-way repeated measure ANOVA and multiple comparisons, $\mathrm{n}=6$ mice/condition. Data shown are representative of three independent experiments.

apoptosis. As shown in Fig. 2A and B, irradiation with various doses resulted in dose-dependent apoptosis in the CNE-2Z tumor cells. A slight induction of apoptosis in the CNE-2Z tumor cells was observed at 2 Gy (14.51\% early apoptosis). Moderate apoptotic induction was observed at 4 Gy (26.13\%), and significant apoptotic induction was observed starting at 6 Gy $(41.46 \%)$ with maximum induction observed at 8 Gy $(61.75 \%)$. To avoid the serious side-effects of a high dose of radiation and in order to leave a window for the observation of combined effect, we chose $4 \mathrm{~Gy}$ as the radiation dose in the in vitro combined treatment with AdVIL-24 gene therapy for CNE-2Z human NPC cells. To examine the combined antitumor effect of AdVIL-24 plus radiotherapy in vitro on the CNE-2Z tumor cells, CNE-2Z human NPC cells were treated with AdVIL-24 (100 MOI), AdV (100 MOI), PBS or 4 Gy alone, or AdVIL-24 (100 MOI) plus 4 Gy. The tumor cell viability was determined daily for 4 days by MTT assay. As shown in Fig. 2C, the combined treatment with AdVIL-24 gene therapy plus 4 Gy radiotherapy additively inhibited the in vitro CNE-2Z tumor cell growth in a time-dependent manner, 

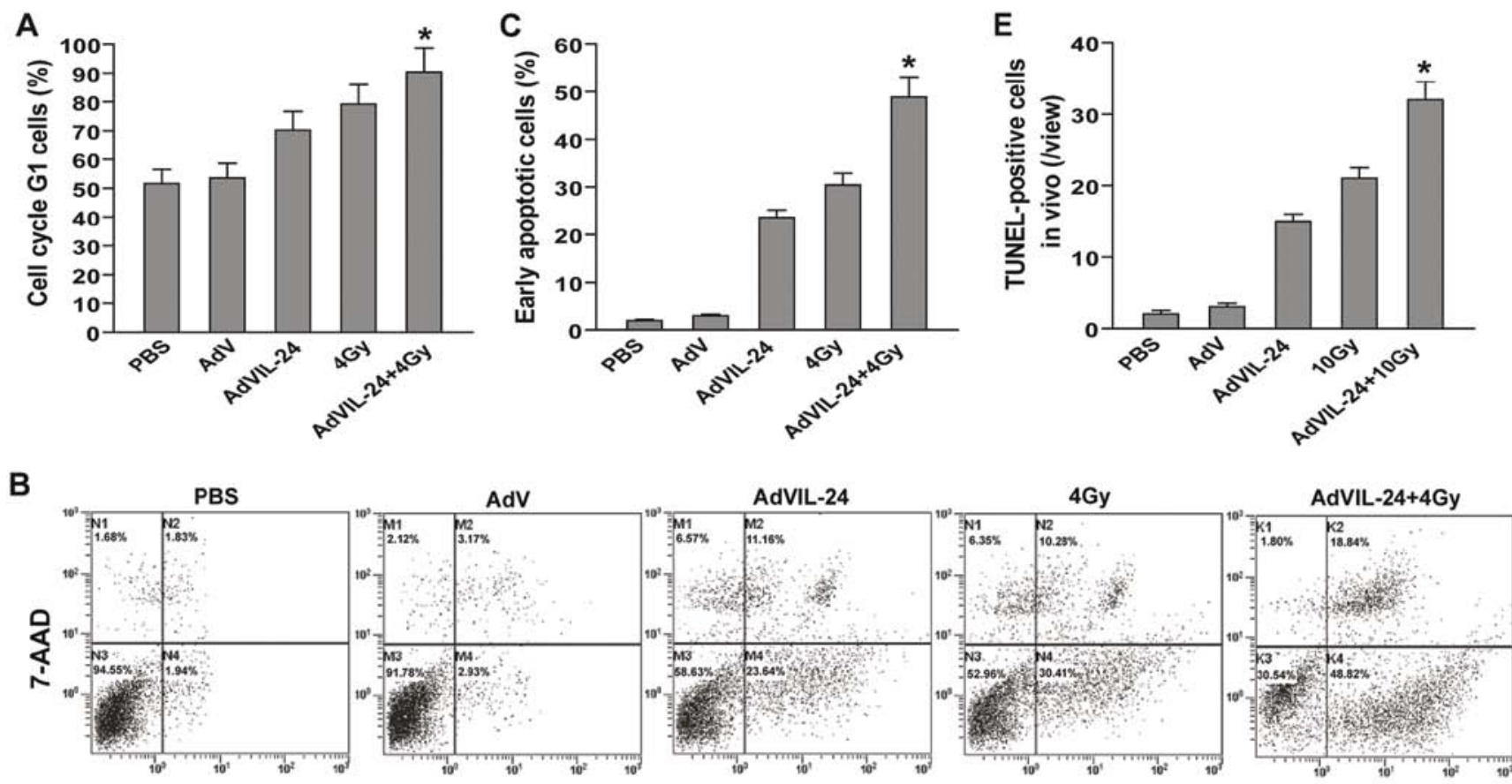

Annexin V-PE

D
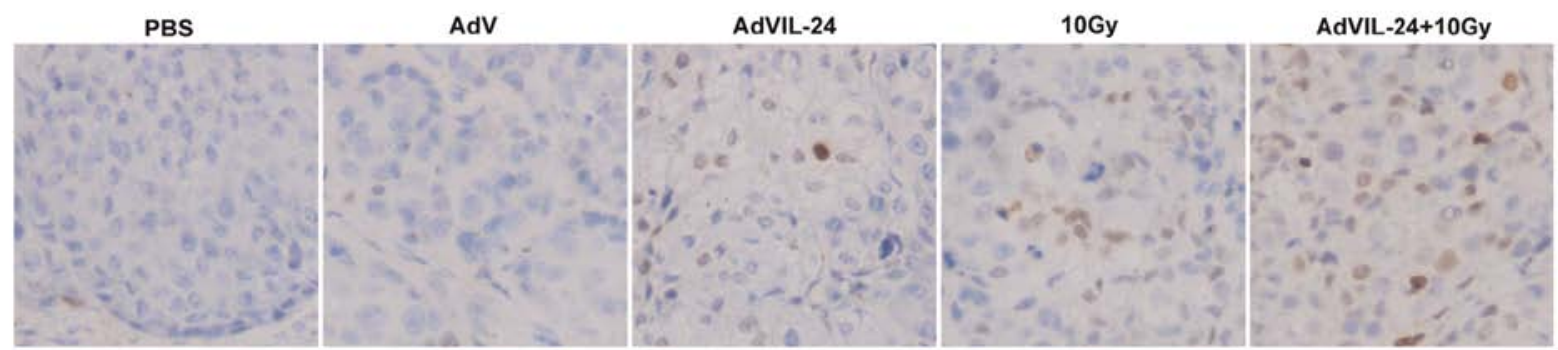

Figure 3. Adenovirus-mediated interleukin-24 (AdVIL-24) plus radiotherapy enhances G1 phase arrest and apoptosis. (A) In vitro cell cycle analysis by flow cytometry. The CNE-2Z human NPC cells were treated with PBS, AdV, AdVIL-24, 4 Gy or AdVIL-24 plus 4 Gy. Forty-eight hours after infection (i.e. $24 \mathrm{~h}$ after irradiation), the tumor cells were harvested, fixed in $70 \%$ cold alcohol, stained with propidium iodide and then analyzed by flow cytometry. The G1 phase cells in the total cell population are presented. " $\mathrm{P}<0.05$ compared with AdVIL-24 and 4 Gy groups; $\mathrm{Q}=0.943$, one-way repeated measure ANOVA and multiple comparisons, $\mathrm{n}=3$ replicates/condition. (B and C) In vitro apoptosis analysis by flow cytometry. The CNE-2Z tumor cells were treated with PBS, AdV, AdVIL-24, 4 Gy or AdVIL-24 plus 4 Gy. (B) Forty-eight hours after infection (i.e. $24 \mathrm{~h}$ after irradiation), the tumor cells were harvested, stained with Annexin V-PE and 7-AAD and then analyzed by flow cytometry. (C) The Annexin V single-positive cells in the total cell population represented early apoptotic cells. " $\mathrm{P}<0.05$ compared with AdVIL-24 and 4 Gy group; $\mathrm{Q}=1.042$, one-way repeated measures ANOVA and multiple comparisons, $\mathrm{n}=3$ replicates/condition. (D and E) In vivo apoptosis analysis by TUNEL assay. (D) Representative immunohistochemical images of TUNEL analysis of the CNE-2Z human NPC subcutaneously xenografted tumors are shown. (E) The TUNEL-positive cells represented in vivo apoptotic cells. "P<0.05 compared with AdVIL-24 and 10 Gy group; $\mathrm{Q}=0.974$, one-way repeated measure ANOVA and multiple comparisons, $\mathrm{n}=6$ mice/condition, $\mathrm{n}=5$ observations/representative section. Data shown are representative of three independent experiments.

compared with the single AdVIL-24- and 4 Gy-treated group $(\mathrm{P}<0.05 ; \mathrm{Q}=0.916$ and 1.050 , at day 3 and 4 after treatment, respectively). The in vitro combined tumor-suppressive effect of AdVIL-24 gene therapy plus ionizing radiotherapy on CNE-2Z human NPC cells was confirmed by clonogenic survival assay (Fig. 2D) $(\mathrm{P}<0.05 ; \mathrm{Q}=1.074)$. To further explore whether the combination of AdVIL-24 plus radiotherapy exerts an in vivo enhanced antitumor effect, the athymic nude mice bearing CNE-2Z human NPC s.c. xenografted tumors were intratumorally injected with AdVIL-24, AdV or PBS alone, or irradiated with 10 Gy alone or in combination with intratumoral injection of AdVIL-24. The tumor growth in vivo was monitored daily, and tumor volume and weight were measured. As shown in Fig. 2E-G, the tumor growth was more significantly retarded in the AdVIL-24 plus 10 Gy group, compared with single AdVIL-24- and 10 Gy-treated groups $\left(\mathrm{P}<0.05 ; \mathrm{Q}_{\text {volume }}=1.099,1.098,0.981,1.127\right.$ and 1.053, at day 6, 8, 10, 12 and 14 after treatment, and $\mathrm{Q}_{\text {weight }}=1.004$, respectively), indicating that the combination treatment with AdVIL-24 plus 10 Gy markedly suppressed CNE-2Z human NPC s.c. xenografted tumor growth in vivo in the athymic nude mice with an overlapping effect.

Alteration in cell cycle distribution and enhanced induction of apoptosis by AdVIL-24 plus radiation. To explore the mechanism by which combination therapy with AdVIL-24 and radiation additively suppresses tumor cell growth, the in vitro cell cycle profiles and apoptosis of the CNE-2Z cells treated with AdVIL-24 or 4 Gy alone, or AdVIL-24 plus 4 Gy for $48 \mathrm{~h}$ were analyzed using PI single staining, and Annexin $\mathrm{V}-\mathrm{PE} / 7-\mathrm{AAD}$ double staining by flow cytometry, respectively. As shown in Fig. 3A, compared with the PBS (51.61\%) and AdV (53.52\%) control group, a significant increase in the G1 phase population was observed in the AdVIL-24 (70.22\%), 
A

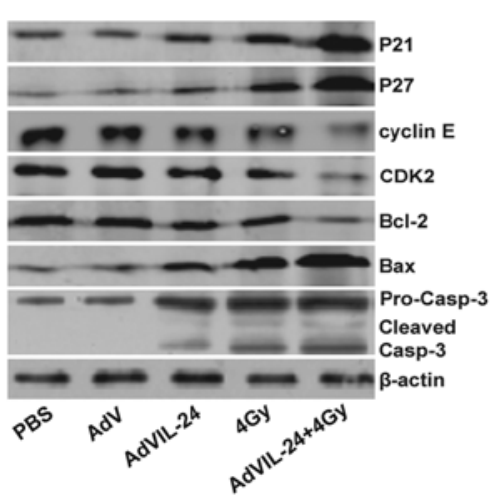

B

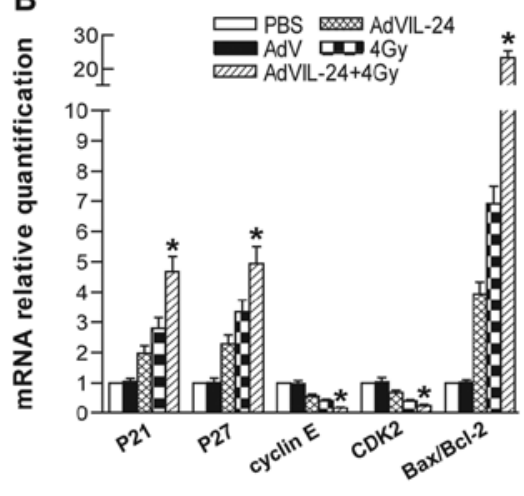

C

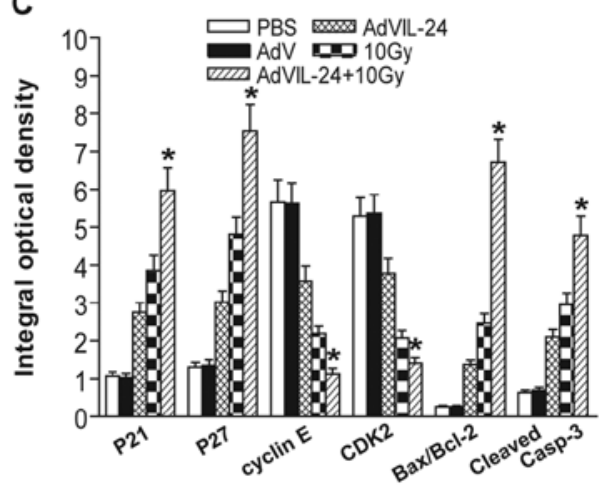

Figure 4. Adenovirus-mediated interleukin-24 (AdVIL-24) plus radiotherapy enhances the upregulation of P21 and P27 cyclin-dependent kinase (CDK) inhibitors and downregulation of cyclin $\mathrm{E}$ and $\mathrm{CDK} 2$ as well as activation of intrinsic apoptosis. (A) Western blot analysis of cell cycle regulatory molecules and apoptosis-related proteins. The total cellular lysates derived from CNE-2Z human NPC cells treated with PBS, AdV, AdVIL-24, 4 Gy or AdVIL-24 plus 4 Gy were subject to western blot analysis using a panel of antibodies specific for P21, P27, cyclin E, CDK2, Bcl-2, Bax, caspase-3 and $\beta$-actin. (B) Real-time quantitative reverse transcription (RT)-PCR analysis of cell cycle regulatory molecules and apoptosis-related proteins. The total cellular RNAs derived from CNE-2Z tumor cells after different treatments were subjected to SYBR-Green I-based real-time quantitative RT-PCR analysis using primer sets specific for $\mathrm{P} 21, \mathrm{P} 27$, cyclin E, CDK2, Bcl-2, Bax and $\beta$-actin. In each case, the transcriptional expression of P21, P27, cyclin E, CDK2 and Bax/Bcl-2 was normalized to the expression level of $\beta$-actin, and the relative change was expressed as a ratio, with 1 being the value for PBS-treated control cells. ${ }^{*} \mathrm{P}<0.05$ compared with the AdVIL-24 and 4 Gy group, $\mathrm{Q}_{\mathrm{P} 21}=0.959, \mathrm{Q}_{\mathrm{P} 27}=0.956, \mathrm{Q}_{\text {cyclin E }}=1.078, \mathrm{Q}_{\mathrm{CDK} 2}=1.046$ and $\mathrm{Q}_{\mathrm{Bax} / \mathrm{Bcl}-2}=0.995$, respectively; one-way repeated measures ANOVA and multiple comparisons, $n=3$ replicates/condition, $n=3$ replicates/sample. (C) Immunohistochemical analysis of cell cycle regulatory molecules and apoptosis-related proteins. The immunostaining intensity of P21, P27, cyclin E, CDK2, Bcl-2, Bax and cleaved caspase-3 was quantified as integral optical density by Image-Pro Plus 6.0 software. ${ }^{*} \mathrm{P}<0.05$ compared with the AdVIL-24 and 10 Gy groups, $\mathrm{Q}_{\mathrm{P} 21}=0.920, \mathrm{Q}_{\mathrm{P} 27}=0.937, \mathrm{Q}_{\mathrm{cyclin} \mathrm{E}}=1.060, \mathrm{Q}_{\mathrm{CDK} 2}=1.019, \mathrm{Q}_{\mathrm{Bax}}$ $\mathrm{Bcl}_{-2}=0.982$ and $\mathrm{Q}_{\text {cleaved caspase- } 3}=0.927$, respectively; one-way repeated measures ANOVA and multiple comparisons, $\mathrm{n}=6 \mathrm{replicates} /$ condition, $\mathrm{n}=5$ observations/ representative section. Data shown are representative of three independent experiments.

4 Gy (79.24\%) and AdVIL-24 plus 4 Gy (90.37\%) groups $(\mathrm{P}<0.05)$. Compared with the single AdVIL-24- and 4 Gy-treated groups, AdVIL-24 plus 4 Gy additively induced CNE-2Z tumor cell cycle G1 phase arrest $(\mathrm{P}<0.05 ; \mathrm{Q}=0.943)$. Furthermore, AdVIL-24 plus radiation induced $48.82 \%$ early apoptosis in CNE-2Z tumor cells, whereas there was only $1.94,2.93,23.64$ and $30.41 \%$ CNE-2Z cells undergoing early apoptosis in the PBS, AdV, AdVIL-24 and 4 Gy groups (Fig. 3B and C). Compared with the single AdVIL-24- and 4 Gy-treated groups, treatment with AdVIL-24 plus 4 Gy more efficiently elicited CNE-2Z tumor cell apoptosis with an overlapping effect $(\mathrm{P}<0.05 ; \mathrm{Q}=1.042)$. To further assess the induction of apoptosis in vivo, we performed a TUNEL assay in the treated and untreated CNE-2Z human NPC s.c. xenografted tumors by immunohistochemistry analysis (Fig. 3D and E). Consistent with the flow cytometric results in vitro, AdVIL-24 plus radiation also had an additive effect on the in vivo apoptosis induction of CNE-2Z human NPC cells s.c. implanted in the athymic nude mice $(\mathrm{P}<0.05 ; \mathrm{Q}=0.974)$.

\section{Enhanced upregulation of P21 and P27 CDK inhibitors} (CDIs) and Bax/Bcl-2 as well as downregulation of cyclin $E$ and CDK2 following treatment with AdVIL-24 plus radiation. To further elucidate the molecular mechanism underlying the antitumor effect mediated by combined therapy of AdVIL-24 plus radiation, the expression levels of cell cycle regulatory molecules such as P21 and P27 CDIs, cyclin E and CDK2 and apoptosis-related proteins including Bcl-2, Bax and caspase-3 in the CNE-2Z cells after different treatments were analyzed by western blotting. As shown in Fig. 4A, the expression of P21, P27, and Bax in the AdVIL-24, 4 Gy and AdVIL-24 plus 4 Gy groups was significantly increased, whereas the expression of cyclin E, CDK2 and Bcl-2 was decreased. In addition, cleavage of caspase-3 was noted in the AdVIL-24, 4 Gy and AdVIL-24 plus 4 Gy groups but not in the PBS and AdV groups. Moreover, the combined treatment of AdVIL-24 plus radiation resulted in an enhanced effect on the upregulation of P21 and P27 CDIs and the ratio of pro-apoptotic to anti-apoptotic molecules Bax/Bcl-2, downregulation of cyclin E and CDK2 and activation of caspase-3. The effect of AdVIL-24 plus radiation on in vitro expression of P21, P27, cyclin E, CDK2 and $\mathrm{Bax} / \mathrm{Bcl}-2$ in CNE-2Z tumor cells and in vivo expression of P21, P27, cyclin E, CDK2, Bax/Bcl-2 and cleaved caspase-3 in CNE-2Z NPC s.c. xenografted tumors was further confirmed by real-time quantitative RT-PCR analysis (Fig. 4B) $(\mathrm{P}<0.05$; $\mathrm{Q}_{\mathrm{P} 21}=0.959, \mathrm{Q}_{\mathrm{P} 27}=0.956, \mathrm{Q}_{\text {cyclinE }}=1.078, \mathrm{Q}_{\mathrm{CDK} 2}=1.046$ and $\mathrm{Q}_{\mathrm{Bax} /}$ $\left.\mathrm{Bcl}_{-2}=0.995\right)$ and immunohistochemistry (Fig. 4C) $(\mathrm{p}<0.05$; $\mathrm{Q}_{\mathrm{P} 21}=0.920, \mathrm{Q}_{\mathrm{P} 27}=0.937, \mathrm{Q}_{\text {cyclin } \mathrm{E}}=1.060, \mathrm{Q}_{\mathrm{CDK} 2}=1.019, \mathrm{Q}_{\mathrm{Bax} /}$ Bcl-2 $=0.982$ and $\mathrm{Q}_{\text {cleaved caspase-3 }}=0.927$ ).

Enhanced reduction of MVD by AdVIL-24 plus radiation. Tumor angiogenesis plays a critical role in cancer progression. To examine the combined effect of AdVIL-24 plus radiation on tumor angiogenesis in vivo, the MVD in CNE-2Z human NPC s.c. xenografted tumors was analyzed by CD34 immunohistochemical analysis. The positive expression of CD34 was mainly presented as brownish yellow or brownish granules in vascular endothelial cells of the CNE-2Z human NPC s.c. xenografted tumors (Fig. 5A). Compared with the PBS and AdV groups, the CD34 expression of tumor vascular endothelial cells in the AdVIL-24, 10 Gy and AdVIL-24 plus 10 Gy groups was weaker or less (Fig. 5A and $\mathrm{B})(\mathrm{P}<0.05)$. In addition, the MVD (Fig. 5C) determined in the AdVIL-24, 10 Gy and AdVIL-24 plus 10 Gy groups was significantly less than that in the PBS and AdV groups $(\mathrm{P}<0.05)$. Moreover, AdVIL-24 gene therapy plus $10 \mathrm{~Gy}$ radiotherapy had an overlapping effect 

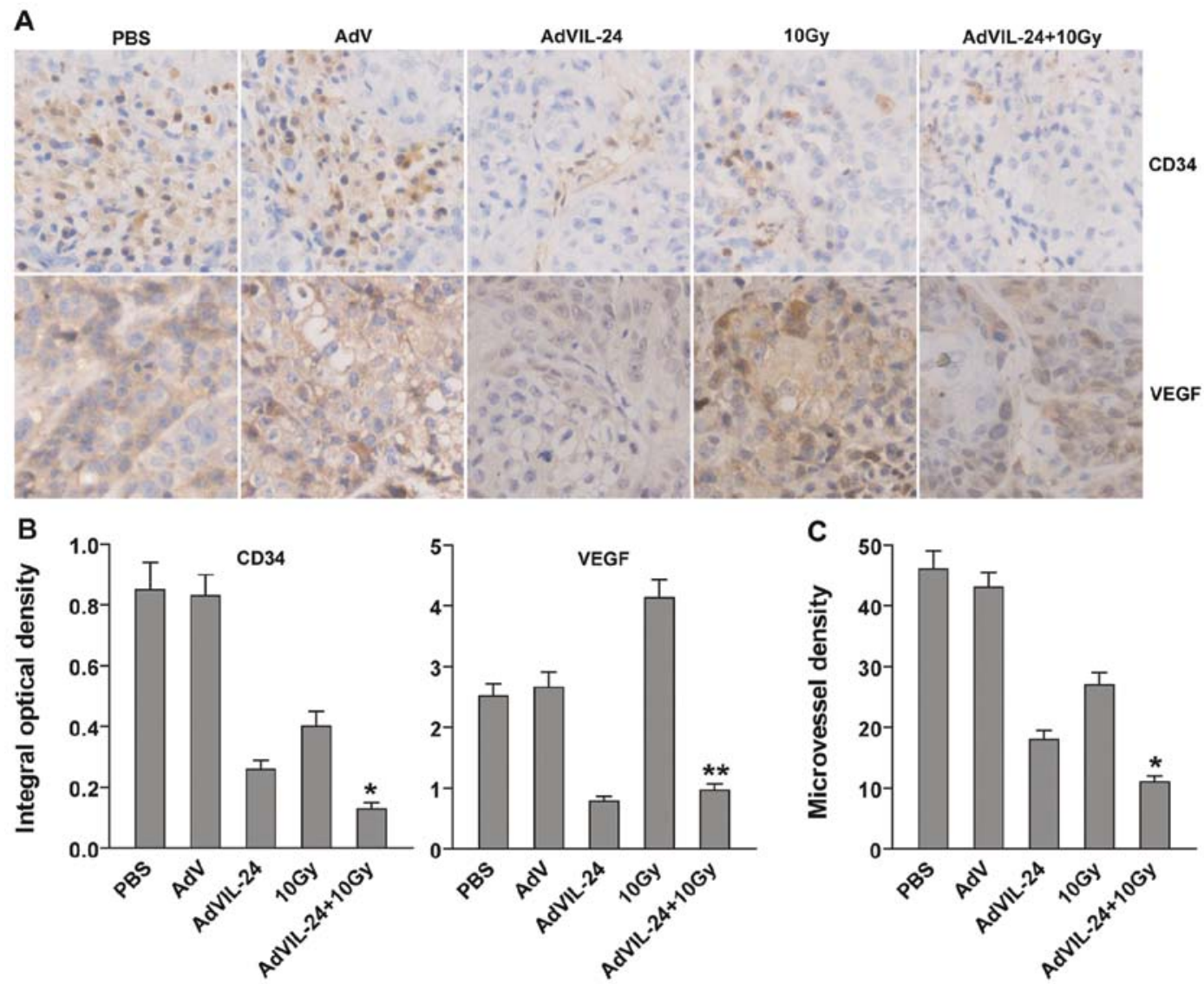

Figure 5. Adenovirus-mediated interleukin-24 (AdVIL-24) plus radiotherapy enhances the reduction in tumor angiogenesis. (A) Representative images of the immunohistochemical detection of CD34 and vascular endothelial growth factor (VEGF) in CNE-2Z human NPC s.c. xenografted tumors. (B) The integral optical density of CD34 and VEGF immunostaining intensity quantified by Image-Pro Plus 6.0 software. ${ }^{*} \mathrm{P}<0.05$ compared with the AdVIL-24 and 10 Gy groups, $\mathrm{Q}=0.990 ;{ }^{* *} \mathrm{P}<0.05$ compared with the $10 \mathrm{~Gy}$ group, respectively; one-way repeated measure ANOVA and multiple comparisons, $\mathrm{n}=6$ replicates/condition, $\mathrm{n}=5$ observations/representative section. (C) The microvessel density in CNE-2Z human NPC xenografted tumors. "P $<0.05$ compared with AdVIL-24 and 10 Gy groups, $\mathrm{Q}=0.988$; one-way repeated measure ANOVA and multiple comparisons, $\mathrm{n}=6$ replicates/condition, $\mathrm{n}=5$ observations/representative section. Data shown are representative of three independent experiments.

on the downregulation of CD34 and the reduction in MVD in the CNE-2Z human NPC s.c. xenografted tumors $(\mathrm{p}<0.05$; $\mathrm{Q}_{\mathrm{CD} 34}=0.990$ and $\mathrm{Q}_{\mathrm{MVD}}=0.988$ ), which may be responsible for the AdVIL-24 and 10 Gy combined therapy-induced enhanced growth suppression of the in vivo CNE-2Z human NPC s.c. xenografted tumors in the athymic nude mice.

AdVIL-24 suppresses radiation-induced expression of VEGF, a pro-angiogenic factor. Tumors can express pro-angiogenic factors, which promote the formation of tumor blood vessels and consequently facilitate tumor growth in vivo. To investigate whether AdVIL-24, radiation, or their combination affectes the production of pro-angiogenic factors, we assessed VEGF expression in vivo in the CNE-2Z human NPC s.c. xenografted tumors by immunohistochemical analysis. As shown in Fig. 5A and B, some constitutive expression of VEGF was apparent in the PBS or AdV groups. AdVIL-24 alone significantly inhibited the expression levels of VEGF $(\mathrm{P}<0.05)$. However, radiation alone substantially enhanced its expression $(\mathrm{P}<0.05)$. Of note, AdVIL-24 potentially blocked radiation-induced enhancement of VEGF expression, indicating that AdVIL-24 gene therapy is capable of impairing the radiation-elicited pro-angiogenic activity via suppressing the production of pro-angiogenic factors such as VEGF.

\section{Discussion}

NPC is a major malignant tumor of the head and neck region, with high a incidence in Southern China and Southeast Asia. Radiotherapy or combination with chemotherapy is currently the mainstay of proven treatment strategies. However, the local recurrence and distant metastasis noted in NPC remains a significant obstacle to therapeutic strategies, which is a main reason for treatment failure. Thus, the development of novel therapeutic approaches for NPC is of paramount importance. Gene therapy is a promising treatment modality for cancer. Preclinical studies and clinical trials have shown that AdV p53 gene therapy alone or combined with ionizing radiotherapy and chemotherapy exerts strong antitumor potential in NPC (18,30-32). Extensive studies have demonstrated that IL-24 can specifically induce apoptosis in a large variety of tumor cells, suppress tumor angiogenesis, stimulate immune responses, promote bystander antitumor activity and enhance radio- and chemo-sensitivity. Therefore, this prompted us to extend previous research and examine the therapeutic effect of AdVIL-24 gene therapy combined with ionizing radiation on NPC. In this study, we found that AdVIL-24 gene therapy combined with ionizing radiotherapy induced enhanced growth suppression, G1 phase arrest and apoptosis in vitro in 
CNE-2Z human NPC cells. Moreover, AdVIL-24 plus radiotherapy also additively inhibited in vivo CNE-2Z human NPC s.c. xenografted tumor growth in athymic nude mice.

Cyclins, CDKs and CDIs are necessary for cell cycle progression. The activities of these cyclin/CDK complexes are negatively regulated by CDIs by directly preventing CDK phosphorylation and inhibiting their activity. P21 and P27 as important members of the CDIs belong to the Cip/Kip family and suppress the activation of cyclin E-CDK2, cyclin D-CDK4 and cyclin A-CDK2 complexes, leading to cell cycle G1 phase arrest $(33,34)$. Initiation of apoptosis induced by irreparable cellular damage is a key mechanism by which ionizing radiation kills cancer cells. Members of the Bcl-2 protein family are known to be key regulators of apoptosis and crucial determinants of cellular fate (35). Bcl-2 is an important anti-apoptotic protein containing four conserved Bcl-2 homology (BH) domains, which heterodimerizes pro-apoptotic protein Bax and its overexpression protects cells from apoptosis induced by different stimuli. The ratio of pro-apoptotic to anti-apoptotic molecules such as Bax/Bcl-2 constitutes a rheostat that sets the threshold of susceptibility to apoptosis for the intrinsic pathway, including pore formation in the mitochondrial outer membrane, loss of mitochondrial integrity and the release of cytochrome $c$ into the cytosol followed by the cleavage of caspase- 9 and -3 (35). Thus, overlapping effect of AdVIL-24 plus radiation on the upregulation of P21 and P27 CDK inhibitors and Bax/Bcl-2 ratio, downregulation of cyclin $\mathrm{E}$ and $\mathrm{CDK} 2$ and activation of caspase-3 may be responsible for the enhanced induction of G1 phase arrest and apoptosis, and consequent additive growth suppression in NPC.

Tumor angiogenesis is a prerequisite for successful tumor growth and formation of metastasis, and is regulated by the balance of tumor-derived pro-angiogenic and anti-angiogenic factors. Thus, tumor angiogenesis is a potential therapeutic target in anticancer therapy. It has been shown that VEGF is a crucial pro-angiogenic factor for tumor angiogenesis (36). Accumulating evidence also suggests that ionizing radiation can induce VEGF production and subsequently facilitate the formation of tumor vessels, leading to tumor regrowth and accelerated metastasis $(37,38)$. Combined therapy with radiotherapy and an angiogenesis inhibitor can override the ionizing radiation-elicited pro-angiogenic activity, thereby exhibiting enhanced antitumor activity. In our study, we demonstrated that AdVIL-24 gene therapy plus ionizing radiotherapy additively inhibited CD34 expression and reduced MVD in CNE-2Z human NPC s.c. xenografted tumors, which may be another important mechanism involved in AdVIL-24 plus radiationmediated in vivo enhanced growth inhibition of CNE-2Z human NPC xenografted tumors in athymic nude mice. In agreement with a previous report (37), ionizing radiation alone abundantly elevated the levels of the pro-angiogenic factor VEGF. Notably, AdVIL-24 efficiently blocked the upregulation of VEGF induced by radiation, likely contributing to the enhanced anti-angiogenic effect.

Taken together, AdVIL-24 gene therapy plus ionizing radiotherapy induced enhanced growth inhibition, cell cycle G1 phase arrest and apoptosis in vitro in CNE-2Z human NPC cells and in vivo in CNE-2Z xenografted tumors s.c. implanted in athymic nude mice. Mechanistically, AdVIL-24 combined with ionizing radiation led to the substantial upregulation of P21 and P27 CDK inhibitors, ratio of pro-apoptotic to anti-apoptotic molecules Bax/Bcl-2 and cleaved caspase-3 as well as downregulation of cyclin $\mathrm{E}$ and $\mathrm{CDK} 2$ in vitro and in vivo in CNE-2Z human NPC cells. Furthermore, AdVIL-24 plus radiation additively reduced the tumor vessel CD34 expression and MVD in vivo. More importantly, AdVIL-24 potentially blocked the radiation-induced enhancement of VEGF. The enhanced antitumor activity against NPC elicited by AdVIL-24 gene therapy combined with ionizing radiotherapy was closely associated with the enhanced induction of G1 phase arrest and apoptosis via the additive modulation of cell cycle regulatory molecules and activation of the intrinsic apoptotic pathway, and the overlapping inhibition of tumor angiogenesis. Thus, our results suggest that AdVIL-24 gene therapy combined with ionizing radiotherapy may be a novel and effective treatment strategy for human NPC.

\section{Acknowledgements}

This research was supported by grants from the National Natural Science Foundation of China (no. 81001016) and the Medicine Research Foundation of the Board of Health of Suzhou City (no. SYS201014).

\section{References}

1. Wei WI and Sham JS: Nasopharyngeal carcinoma. Lancet 365: 2041-2054, 2005

2. Young LS and Rickinson AB: Epstein-Barr virus: 40 years on. Nat Rev Cancer 4: 757-768, 2004.

3. Spano JP, Busson P, Atlan D, Bourhis J, Pignon JP, Esteban C and Armand JP: Nasopharyngeal carcinomas: an update. Eur J Cancer 39: 2121-2135, 2003.

4. Yu MC and Yuan JM: Epidemiology of nasopharyngeal carcinoma. Semin Cancer Biol 12: 421-429, 2002.

5. Guigay J: Advances in nasopharyngeal carcinoma. Curr Opin Oncol 20: 264-269, 2008.

6. Lee AW, Yau TK, Wong DH, et al: Treatment of stage IV(A-B) nasopharyngeal carcinoma by induction-concurrent chemoradiotherapy and accelerated fractionation. Int J Radiat Oncol Biol Phys 63: 1331-1338, 2005.

7. Le QT, Tate D, Koong A, et al: Improved local control with stereotactic radiosurgical boost in patients with nasopharyngeal carcinoma. Int J Radiat Oncol Biol Phys 56: 1046-1054, 2003.

8. Lee AW, Sze WM, Au JS, et al: Treatment results for nasopharyngeal carcinoma in the modern era: the Hong Kong experience. Int J Radiat Oncol Biol Phys 61: 1107-1116, 2005.

9. Sauane M, Gopalkrishnan RV, Sarkar D, et al: MDA-7/IL-24: novel cancer growth suppressing and apoptosis inducing cytokine. Cytokine Growth Factor Rev 14: 35-51, 2003.

10. Fisher PB: Is mda-7/IL-24 a 'magic bullet' for cancer? Cancer Res 65: 10128-10138, 2005.

11. Ramesh R, Mhashilkar AM, Tanaka F, et al: Melanoma differentiation-associated gene 7/interleukin (IL)-24 is a novel ligand that regulates angiogenesis via the IL-22 receptor. Cancer Res 63: 5105-5113, 2003.

12. Nishikawa T, Ramesh R, Munshi A, Chada S and Meyn RE: Adenovirus-mediated mda-7 (IL24) gene therapy suppresses angiogenesis and sensitizes NSCLC xenograft tumors to radiation. Mol Ther 9: 818-828, 2004.

13. Caudell EG, Mumm JB, Poindexter N, et al: The protein product of the tumor suppressor gene, melanoma differentiationassociated gene 7, exhibits immunostimulatory activity and is designated IL-24. J Immunol 168: 6041-6046, 2002.

14. Ramesh R, Ito I, Gopalan B, Saito Y, Mhashilkar AM and Chada S: Ectopic production of MDA-7/IL-24 inhibits invasion and migration of human lung cancer cells. Mol Ther 9: 510-518, 2004.

15. Park MA, Yacoub A, Sarkar D, et al: PERK-dependent regulation of MDA-7/IL-24-induced autophagy in primary human glioma cells. Autophagy 4: 513-515, 2008. 
16. Yacoub A, Hamed HA, Allegood J, et al: PERK-dependent regulation of ceramide synthase 6 and thioredoxin play a key role in mda-7/IL-24-induced killing of primary human glioblastoma multiforme cells. Cancer Res 70: 1120-1129, 2010.

17. Hughes J, Alusi G and Wang Y: Gene therapy and nasopharyngeal carcinoma. Rhinology 50: 115-121, 2012.

18. Li JH, Huang D, Sun BF, Zhang X, Middeldorp J, Klamut H and Liu FF: Efficacy of ionizing radiation combined with adenoviral p53 therapy in EBV-positive nasopharyngeal carcinoma. Int J Cancer 87: 606-610, 2000.

19. Xie Y, Sheng W, Miao J, Xiang J and Yang J: Enhanced antitumor activity by combining an adenovirus harboring ING4 with cisplatin for hepatocarcinoma cells. Cancer Gene Ther 18 : 176-188, 2011.

20. Su ZZ, Lebedeva IV, Sarkar D, et al: Ionizing radiation enhances therapeutic activity of mda-7/IL-24: overcoming radiation- and mda-7/IL-24-resistance in prostate cancer cells overexpressing the antiapoptotic proteins bcl-xL or bcl-2. Oncogene 25 : 2339-2348, 2006.

21. Emdad L, Sarkar D, Lebedeva IV, et al: Ionizing radiation enhances adenoviral vector expressing mda-7/IL-24-mediated apoptosis in human ovarian cancer. J Cell Physiol 208: 298-306, 2006.

22. Liu J, Sheng W, Xie Y, Shan Y, Miao J, Xiang J and Yang J: The in vitro and in vivo antitumor activity of adenovirus-mediated interleukin-24 expression for laryngocarcinoma. Cancer Biother Radiopharm 25: 29-38, 2010.

23. Wang X, Ye Z, Zhong J, Xiang J and Yang J: Adenovirus-mediated Il-24 expression suppresses hepatocellular carcinoma growth via induction of cell apoptosis and cycling arrest and reduction of angiogenesis. Cancer Biother Radiopharm 22: 56-63, 2007.

24. Xie Y, Lv H, Sheng W, Miao J, Xiang J and Yang J: Synergistic tumor suppression by adenovirus-mediated inhibitor of growth 4 and interleukin-24 gene cotransfer in hepatocarcinoma cells. Cancer Biother Radiopharm 26: 681-695, 2011.

25. Livak KJ and Schmittgen TD: Analysis of relative gene expression data using real-time quantitative PCR and the 2(-Delta Delta C(T)) method. Methods 25: 402-408, 2001.

26. Schmittgen TD and Livak KJ: Analyzing real-time PCR data by the comparative C(T) method. Nat Protoc 3: 1101-1108, 2008 .
27. Madhusoodhanan R, Natarajan M, Veeraraghavan J, Herman TS and Aravindan N: NFkappaB activity and transcriptional responses in human breast adenocarcinoma cells after single and fractionated irradiation. Cancer Biol Ther 8: 765-773, 2009.

28. Weidner N: Current pathologic methods for measuring intratumoral microvessel density within breast carcinoma and other solid tumors. Breast Cancer Res Treat 36: 169-180, 1995.

29. Wang W, Qin SK, Chen BA and Chen HY: Experimental study on antitumor effect of arsenic trioxide in combination with cisplatin or doxorubicin on hepatocellular carcinoma. World J Gastroenterol 7: 702-705, 2001.

30. Li JH, Li P, Klamut H and Liu FF: Cytotoxic effects of Ad5CMVp53 expression in two human nasopharyngeal carcinoma cell lines. Clin Cancer Res 3: 507-514, 1997.

31. Pan JJ, Zhang SW, Chen CB, et al: Effect of recombinant adenovirus-p53 combined with radiotherapy on long-term prognosis of advanced nasopharyngeal carcinoma. J Clin Oncol 27: 799-804, 2009.

32. Weinrib L, Li JH, Donovan J, Huang D and Liu FF: Cisplatin chemotherapy plus adenoviral p53 gene therapy in EBV-positive and -negative nasopharyngeal carcinoma. Cancer Gene Ther 8: 352-360, 2001.

33. Harper JW, Adami GR, Wei N, Keyomarsi K and Elledge SJ: The p21 Cdk-interacting protein Cip1 is a potent inhibitor of G1 cyclin-dependent kinases. Cell 75: 805-816, 1993.

34. Toyoshima $\mathrm{H}$ and Hunter T: p27, a novel inhibitor of G1 cyclinCdk protein kinase activity, is related to p21. Cell 78: 67-74, 1994.

35. Danial NN and Korsmeyer SJ: Cell death: critical control points. Cell 116: 205-219, 2004

36. Dvorak HF: Vascular permeability factor/vascular endothelial growth factor: a critical cytokine in tumor angiogenesis and a potential target for diagnosis and therapy. J Clin Oncol 20: 4368-4380, 2002.

37. Moeller BJ, Cao Y, Li CY and Dewhirst MW: Radiation activates HIF-1 to regulate vascular radiosensitivity in tumors: role of reoxygenation, free radicals, and stress granules. Cancer Cell 5: 429-441, 2004

38. Camphausen K, Moses MA, Beecken WD, Khan MK, Folkman J and O'Reilly MS: Radiation therapy to a primary tumor accelerates metastatic growth in mice. Cancer Res 61: 2207-2211, 2001 